
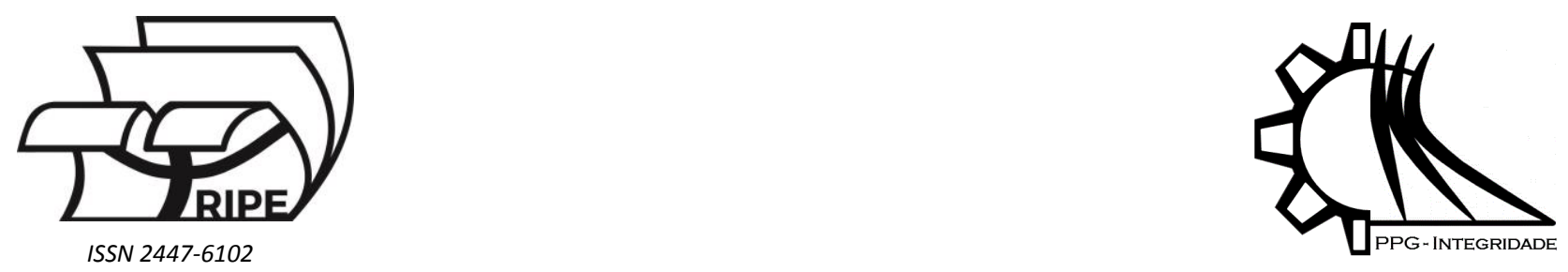

Article

\title{
Estudo numérico das propriedades viscoelásticas no domínio do tempo em misturas asfálticas do tipo CPA
}

\author{
García, L.M. ${ }^{1}$, Farias, M.M . ${ }^{2}$, Recarey, C.M. ${ }^{3}$ and Souza, M.V.R. ${ }^{4, *}$ \\ 1 Universidade Federal de Goiás (UFG); liomedina83@gmail.com \\ 2 Universidade de Brasília (UnB); mmuniz94@gmail.com \\ 3 Universidad Central “Marta Abreu” de las Villas (UCLV); carecarey@gmail.com \\ 4 Universidade Federal de Tocantins (UFT); marcus.vrs21@gmail.com \\ Correspondence: liomedina83@gmail.com;
}

Received: 14/12/2018; Accepted: 05/01/2019; Published: 06/02/2019

\begin{abstract}
Resumo: $\mathrm{O}$ artigo apresenta as simulações numéricas do ensaio de fluência estática (Creep Compliance) para várias misturas asfálticas tipo CPA (camada porosa de atrito). Os ensaios de fluência estática foram realizados a $25^{\circ} \mathrm{C}$ e resultam nos parâmetros constitutivos representados pela série de Prony. Os parâmetros constitutivos viscoelásticos são obtidos a partir das relações entre as funções no domínio do tempo: a função fluência e o modulo de Relaxação. Para a modelagem numérica foi utilizado o método dos elementos finitos e são modelados em 3D corpos de prova com dimensões aproximadas de 66 a $62 \mathrm{~mm}$ de altura e $102 \mathrm{~mm}$ de diâmetro. Para realizar a modelagem numérica foi utilizado o elemento finito C3D20R, com integração reduzida, já que baseado em estudos de convergência anteriores mostrou resultados mais acurados para analises de problemas similares. Foram determinadas dois conjuntos de parâmetros e comparados os resultados de simulações numéricas em várias misturas asfálticas. Os resultados mostram que para as misturas estudadas a escolha do método para determinar os parâmetros constitutivos tem pouca influência nos resultados numéricos.
\end{abstract}

Palavras-chave: Série de Prony, propriedades viscoelásticas, mistura asfáltica

\section{Numerical study of time dependent viscoelastic properties in porous asphalt mixes, known as CPA.}

\begin{abstract}
This article presents the numerical simulations of the static creep test (Creep Compliance) for several asphalt mixtures of the CPA type (Porous Asphalt Layer). The static creep tests was made at $25^{\circ} \mathrm{C}$ resulting in the constitutive parameters represented by the Prony series. The viscoelastic constitutive parameters are obtained from the relationships between time-dependent functions: the compliance function and the relaxation modulus. The numerical models were made with the finite element method and the samples are modeled in 3D with approximate dimensions of 66 to $62 \mathrm{~mm}$ in height and $102 \mathrm{~mm}$ in diameter. To make the numerical models, the C3D20R element is used, with reduced integration, since, based on previous studies, it showed more precise results in the analysis of similar problems. Two groups of constitutive parameters were determined and the results of the numerical simulations for several mixtures compared. The results show that for the studied mixtures the choice of the method to determine the constitutive parameters has little influence on the numerical results.
\end{abstract}

Keywords: Prony series, viscoelastic properties, asphalt mix 


\section{Introdução}

As misturas asfálticas normalmente são compostos por agregados de várias granulometrias e um ligante (CAP Cimento Asfáltico de Petróleo), obtido da destilação do petróleo. A mistura dos agregados com o ligante pode ser realizada a quente ou frio em uma usina de asfalto. Várias pesquisas como as de Evangelista Jr. et al. (2005), Evangelista Jr. (2006), Collop et al. (2007), Silva et al. (2008) e (Y.-R. Kim, 2013) mostram a importância de considerar o comportamento viscoelástico das misturas asfálticas, devido ao efeito do comportamento do ligante no material compósito. Segundo Amaral (2000), a aplicação da hipótese de comportamento elástico linear é considerada aceitável, quando o tempo de duração do carregamento for relativamente baixo e a temperatura de serviço do pavimento for relativamente baixa (inferior a $25^{\circ} \mathrm{C}$ ). Quando a temperatura de serviço do pavimento for relativamente alta, a parcela elástica das deformações torna-se desprezível, principalmente sob a ação de cargas estáticas de longa duração, sendo necessária a adoção de modelos mais complexos.

\subsection{Viscoelasticidade linear em misturas asfálticas.}

As principais funções que podem ser utilizadas para caracterizar o comportamento viscoelástico linear das misturas asfálticas são o módulo de relaxação $\mathrm{E}(\mathrm{t})$, creep compliance $\mathrm{D}(\mathrm{t})$, o módulo complexo $\mathrm{G}^{*}$ (Y. R. Kim, 2009) e ângulo de fase $\rho$. De acordo com os autores Souza \& Soares (2002) e Kim (2009), essas propriedades são obtidas a partir de duas formas de carregamento diferentes: carregamento harmônico e carregamento constante. Para ligantes asfálticos utiliza-se geralmente o carregamento harmônico para o qual o material é caracterizado pelo módulo complexo $G^{*}$ e o ângulo de fase $\delta$. Para misturas asfálticas, no entanto, é comum a utilização de carregamento constante, sendo o material caracterizado pela função fluência no tempo (creep compliance), definida como:

$$
D(t)=\frac{\varepsilon(t)}{\sigma}
$$

$D(t)$ : função fluência;

$\varepsilon(\mathrm{t})$ : deformação em função do tempo; e

$\sigma:$ tensão constante aplicada

As características viscoelásticas também são apresentadas pela função deformação no tempo (módulo de relaxação), dada pela equação:

$$
E(t)=\frac{\sigma(t)}{\varepsilon}
$$

$\mathrm{E}(\mathrm{t})$ :Módulo de Relaxação;

$\sigma(t)$ : tensão em função do tempo; e

$\varepsilon$ : deformação constante aplicada

Estas relações de tensão-deformação também podem ser expressadas com um operador diferencial baseado em modelos mecânicos que consistem em molas lineares e amortecedores (Park \& Schapery, 1999). O modelo generalizado de Maxwell é formado por uma mola e $n$ elementos Maxwell conectados em paralelo 0 módulo de relaxação é dado pela equação conhecida como série de Prony ou Dirichlet:

$$
\boldsymbol{E}(\boldsymbol{t})=\boldsymbol{E}_{\infty}+\sum_{i=1}^{n} \boldsymbol{E}_{i}^{-\left(\frac{t}{\boldsymbol{\rho}_{i}}\right)}
$$

$\mathrm{E}(\mathrm{t})$ : Módulo de Relaxação

$\mathrm{E}_{\infty}$ : Termo independente da série Prony

$E_{i}$ e $\rho_{i}$ : Termos dependentes da série Prony

Para representar a função fluência é usado comumente o modelo generalizado de Kelvin ou modelo de Voigt, o qual consiste em uma mola, um amortecedor e $n$ elementos Voigt conectados em série: 


$$
\boldsymbol{D}(\boldsymbol{t})=\boldsymbol{D}_{0}+\sum_{i=1}^{n} \boldsymbol{D}_{i}\left[1-\boldsymbol{e}^{-\left(\frac{\boldsymbol{t}}{\tau_{i}}\right)}\right]
$$

$D(t)$ : Função Fluência

$D_{0}$ : Termo independente da série Prony

$D_{i}$ e $\boldsymbol{\tau}_{\mathbf{i}}$ : Termos dependentes da série Prony

$\mathrm{n}$ : Termos da série Prony

\subsection{Ajuste de dados experimentais com séries de Prony}

Existem vários métodos para ajustar as séries de Prony. Entre os métodos comumente usados, encontra-se o Método da Colocação desenvolvido por Schapery (1961), segundo Huang (1993), no qual para valores arbitrados de tempos de relaxação ou retardação $\left(\boldsymbol{\tau}_{\mathbf{i}}\right.$ ou $\left.\rho_{i}\right)$ são calculados valores de $D_{i}$ e $E_{i}$ por meio da resolução de um sistema de equações.

O tamanho da série de Prony é determinado pela escala da variável tempo " $\mathrm{t}$ " devido à influência somente de duas décadas logarítmicas de cada termo da série. Visando determinar a quantidade de termos da série, calcula-se a margem de variação de " $\mathrm{t}$ ", subtrai-se entre as potências de dez do maior e do menor tempo em notação científica e acrescenta-se um (01) ao resultado (Sousa \& Soares, 2007).

\subsection{Interconversão da série de Prony}

Vários métodos aproximados de interconversão entre as funções de relaxamento e de fluência estão disponíveis na literatura. Park \& Kim (1999) e Kim (2009) referem-se, cronologicamente, às principais relações entre as propriedades viscoelásticas no domínio do tempo. A relação mais simples é a Quase Elástica, $\mathrm{E}(\mathrm{t}) \mathrm{D}(\mathrm{t}) \cong 1$, para $\mathrm{t}$ $>0$. Existem outras relações para interconverter estas propriedades e são referidas por Park \& Kim (1999) e Kim (2009). No trabalho de Park \& Schapery (1999) após uma dedução formal e realizando manipulações algébricas, chega-se à equação de interconversão, que permite obter a Função Fluência $D(t)$ a partir do Módulo de Relaxação $E(t)$ :

$$
\begin{aligned}
{\left.[A]\{D\}=\{B\}_{\text {ou }} A_{k j} D_{j}=B_{k} \quad \text { (Somado em } \mathrm{j} ; \mathrm{j}=1, \ldots, \mathrm{N} ; \mathrm{k}=1, \ldots, \mathrm{N}\right) } \\
A_{e}\left[1-e^{-\left(\frac{t_{k}}{\tau_{j}}\right)}\right]+\sum_{i=1}^{N} \frac{\rho_{i} E_{i}}{\rho_{i}-t_{k}}\left[e^{-\left(\frac{t_{k}}{\rho_{i}}\right)}-e^{\left.-\left(\frac{t_{k}}{\tau_{j}}\right)\right]} \cdot \text { se } \rho_{\mathrm{i}} \neq \tau_{\mathrm{j}}\right. \\
\text { ou }\left[\begin{array}{l}
A_{e}\left[1-e^{-\left(\frac{t_{k}}{\tau_{j}}\right)}\right]+\sum_{i=1}^{N} \frac{t_{k} E_{i}}{\tau_{j}}\left[e^{-} \text {se } \rho_{\mathrm{i}}=\tau_{\mathrm{j}}\right.
\end{array}\right.
\end{aligned}
$$

e $B_{k}=1-\left[E_{e}+\sum_{i=1}^{N} E_{i} e^{-\left(\frac{t_{k}}{\rho_{i}}\right)}\right] /\left(E_{e}+\sum_{i=1}^{N} E_{i}\right)$ 
Para o caso inverso, obter a função $E(t)$ a partir de dados experimentais da função fluência $D(t)$, a equação de

interconversão resulta na equação

$$
A_{k i}=\left\{\begin{array}{l}
-D_{0}\left(1-e^{-\frac{t_{k}}{\rho_{j}}}\right)+\sum_{i=1}^{N}-D_{j}\left(1-e^{-\frac{t_{k}}{\rho_{i}}}\right)+\sum_{i=1}^{N} \frac{\tau_{j} D_{j}}{\tau_{j}-\rho_{i}}\left(e^{-\frac{t_{k}}{\tau_{j}}}-e^{-\frac{t_{k}}{\rho_{i}}}\right) \\
-D_{0}\left(1-e^{-\frac{t_{k}}{\rho_{j}}}\right)+\sum_{i=1}^{N}-D_{j}\left(1-e^{-\frac{t_{k}}{\rho_{i}}}\right)+\sum_{i=1}^{N} \frac{D_{j} t_{k}}{\rho_{i}} e^{-\frac{t_{k}}{\rho_{i}}}
\end{array}\right.
$$

(8). Este mesmo método com pequenas modificações é usado também por Fernández et al. (2009, 2011), nas análises das propriedades viscoelásticas lineares do polímero polimetil metacrilato.

$$
\begin{aligned}
& {[A]\{E\}=\{B\} \text { ou } A_{k i} E_{i}=B_{k} \quad(\text { Soma em i; i }=1, \ldots, \mathrm{N} ; \mathrm{k}=1, \ldots, \mathrm{N})} \\
& A_{k i}=\left\{\begin{array}{l}
-D_{0}\left(1-e^{-\frac{t_{k}}{\rho_{j}}}\right)+\sum_{i=1}^{N}-D_{j}\left(1-e^{-\frac{t_{k}}{\rho_{i}}}\right)+\sum_{i=1}^{N} \frac{\tau_{j} D_{j}}{\tau_{j}-\rho_{i}}\left(e^{-\frac{t_{k}}{\tau_{j}}}-e^{-\frac{t_{k}}{\rho_{i}}}\right) \quad \text { se } \rho_{\mathrm{i}} \neq \tau_{\mathrm{j}} \\
-D_{0}\left(1-e^{-\frac{t_{k}}{\rho_{j}}}\right)+\sum_{i=1}^{N}-D_{j}\left(1-e^{-\frac{t_{k}}{\rho_{i}}}\right)+\sum_{i=1}^{N} \frac{D_{j} t_{k}}{\rho_{i}} e^{-\frac{t_{k}}{\rho_{i}}} \text { se } \rho_{\mathrm{i}}=\tau_{\mathrm{j}}
\end{array}\right. \\
& B_{k}=1-\left[D_{0}+\sum_{j=1}^{N} D_{j}\left(1-e^{-\frac{t_{k}}{\tau_{j}}}\right)\right] / D_{0}
\end{aligned}
$$

De acordo com Silva (2009), essas equações resultam na resolução de um sistema de equações lineares, as quais constituem uma vantagem do ponto de vista da implementação computacional. Por esse motivo, vários pesquisadores criaram ferramentas computacionais que permitem fazer o ajuste da série de Prony e a interconversão entre as propriedades dos materiais viscoelásticos. O Software VISCOlab ${ }^{\mathrm{Tm}}$ é uma ferramenta desenvolvida pela empresa Multimechanics para facilitar o tratamento de propriedades vicoelásticas lineares de materiais. Permite o ajuste das funções baseando-se nas séries de Prony e pelo método da colocação. Além disso, permite realizar a interconversão de funções a partir de dados experimentais.

\section{Materiais e métodos}

Com o objetivo de determinar os parâmetros constitutivos viscoelásticos são analisadas várias misturas asfálticas do tipo CPA (Camada Porosa de Atrito). Os resultados experimentais utilizados nestas análises encontram-se descritos no trabalho de Souza (2010). Nesta pesquisa é estudado o desempenho mecânico de concretos asfálticos do tipo CPA, produzidos usando ligante asfáltico CAP 50-70 sem modificar e modificado com fibras sintéticas, e agregados calcários dolomíticos.

O ensaio de função fluência $D(t)$ é usado para a caracterização viscoelástica das misturas asfálticas devido a sua relativa simplicidade. O ensaio de fluência por compressão uniaxial estática consiste, em linhas gerais, na aplicação de cargas de compressão estática e de valor constante, em corpos de prova, por um período de tempo predeterminado sob condições de temperatura padronizada, mantida constante ao longo do ensaio (Amaral, 2000). O ensaio de fluência estática utilizado por Souza, (2010) sugere a aplicação de uma pré-carga de no mínimo 5\% da carga a ser utilizada no ensaio. Nesta pesquisa a pré-carga correspondeu a $5 \mathrm{kPa}$ aplicada por 10 segundos. Os ensaios foram realizados a $25 \pm 0,5$ ㅇ C por meio de uma carga estática, com uma tensão aplicada de $0,1 \mathrm{MPa}\left(1 \mathrm{kgf} / \mathrm{cm}^{2}\right)$ durante uma hora. Na sequência o corpo de prova permaneceu durante 15 minutos em fase de descarregamento com objetivo de verificar o retorno da deformação viscosa da mistura e consequentemente poder avaliar somente a parcela permanente dos deslocamentos.

\subsection{Descrição do modelo numérico.}


Os modelos numéricos utilizados visam descrever o comportamento do material viscoelástico no domínio do tempo a partir da simulação do ensaio de fluência estática considerando as particularidades próprias do problema. Para atingir este objetivo, foi utilizado o método dos elementos finitos e são modelados em 3D corpos de prova com dimensões aproximadas de 66 a $62 \mathrm{~mm}$ de altura e $102 \mathrm{~mm}$ de diâmetro. Empregaram-se basicamente duas abordagens para modelar o mesmo fenômeno e estabelecer comparações. Foi modelado o mesmo ensaio como um corpo homogêneo, mudando apenas os parâmetros constitutivos em função do tipo de relação entre $D(t)$ e $E(t)$.

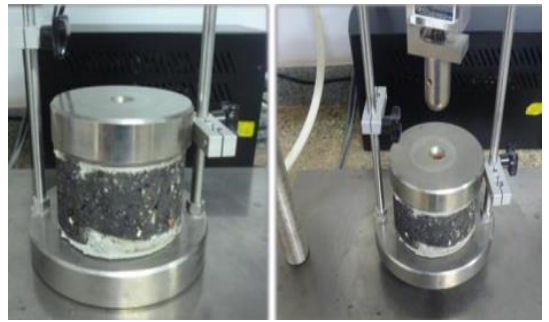

a)

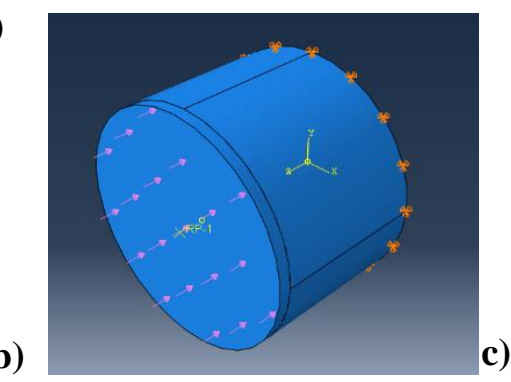

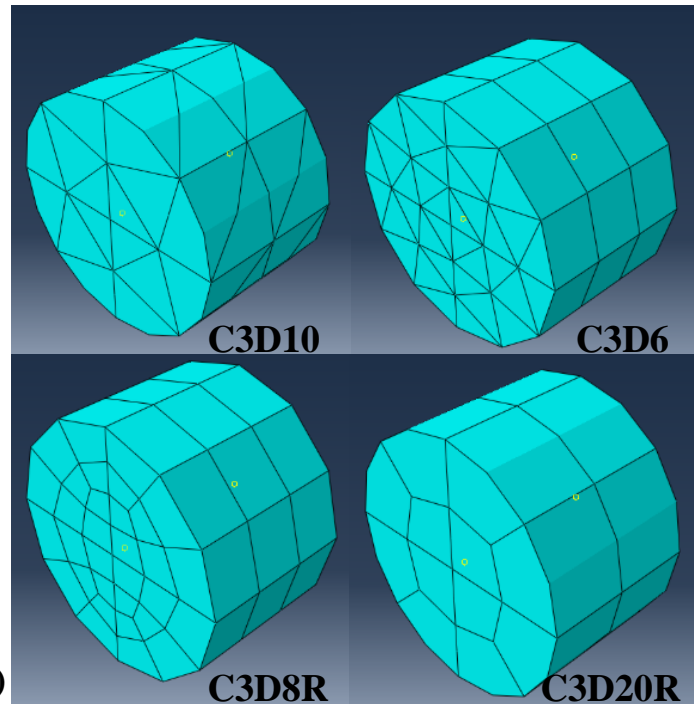

Figura 1. a) Ensaio Creep estático. b) Modelo numérico. c) Tipo e distribuição de elementos usados no estudo de sensibilidade de malhado.

Na Figura 1 Figura 1. a) Ensaio Creep estático. b) Modelo numérico. c) Tipo e distribuição de elementos usados no estudo de sensibilidade de malhado.a), é apresentado o corpo de prova pronto para executar o ensaio real. Na Fig. 1 b), é mostrado o modelo numérico com as simplificações explicadas acima. Na Fig. 1 c), são apresentados os diferentes tipos de elementos finitos usados para realizar o estudo de convergência numérica da malha. 0 modelo C3D10 usa elementos em cunha tridimensional com 10 nós; o elemento C3D6 usa cunha tridimensional com 6 nós; os modelos C3D8 e C3D20 usam elementos do tipo paralelepípedo com 8 e 20 nós, respectivamente. No trabalho de Rodrigues, García, Muniz, e Rodrigues (2016) foi realizado um estudo de convergência para analises de problemas similares e foi definido que resultados mais acurados são obtidos com elementos do C3D20R, com integração reduzida.

\subsection{Parâmetros constitutivos e a série de Prony.}

A partir da função fluência $D(t)$ são determinadas as respectivas séries de Prony para o módulo de relaxação $E(t)$. No primeiro caso é aplicada a relação quase elástica e é determinada a série de Prony para $\mathrm{E}(\mathrm{t})$ aplicando o Método da Colocação Mínimos Quadrados Linear (MQL) descrito por Silva, (2009). No segundo caso e com a ajuda do software VISCOlab $^{\text {TM }}$ os parâmetros da série são determinados utilizando o procedimento da equação

$$
A_{k i}=\left\{\begin{array}{l}
-D_{0}\left(1-e^{-\frac{t_{k}}{\rho_{j}}}\right)+\sum_{i=1}^{N}-D_{j}\left(1-e^{-\frac{t_{k}}{\rho_{i}}}\right)+\sum_{i=1}^{N} \frac{\tau_{j} D_{j}}{\tau_{j}-\rho_{i}}\left(e^{-\frac{t_{k}}{\tau_{j}}}-e^{-\frac{t_{k}}{\rho_{i}}}\right) \\
-D_{0}\left(1-e^{-\frac{t_{k}}{\rho_{j}}}\right)+\sum_{i=1}^{N}-D_{j}\left(1-e^{-\frac{t_{k}}{\rho_{i}}}\right)+\sum_{i=1}^{N} \frac{D_{j} t_{k}}{\rho_{i}} e^{-\frac{t_{k}}{\rho_{i}}}
\end{array}\right.
$$

dois casos o tamanho da série será de quatro elementos levando em consideração os critérios expostos no item 1.2. Foram estabelecidas comparações entre os resultados numéricos e os valores experimentais visando obter a influência do método de interconversão nos resultados.

Considerando que o material para esta pesquisa tem um comportamento viscoelástico linear isotrópico, de acordo com Brinson \& Brinson (2008), as relações são análogas às de materiais linear-elásticos isotrópicos: 


$$
G(t)=\frac{E(t)}{2(1+v)} \quad K(t)=\frac{E(t)}{3(1-2 v)}
$$

$\mathrm{G}(\mathrm{t})$ : Módulo de Cisalhamento no tempo

$\mathrm{K}(\mathrm{t})$ : Módulo de volumétrico no tempo

$v$ : Coeficiente de Poisson

No software ABAQUS, o formato de entrada desses parâmetros do modelo viscoelástico é ligeiramente diferente, e baseia-se em uma série de Prony normalizada para a função de módulo de cisalhamento no tempo Kong e Yuan (2010). O conjunto de dados característicos do material é estabelecido através da associação de suas características elásticas e viscoelásticas. Para o Módulo de Young há opção de entrada com o módulo elástico em longo prazo ou o módulo instantâneo. Para esta análise foi adotado o módulo a longo prazo, dado pelo parâmetro

independente da Série de Prony referida na Eq.

$$
\boldsymbol{E}(\boldsymbol{t})=\boldsymbol{E}_{\infty}+\sum_{i=1}^{n} \boldsymbol{E}_{i}^{-\left(\frac{\boldsymbol{t}}{\boldsymbol{\rho}_{i}}\right)}
$$

(3).

É preciso ressaltar que algumas simplificações foram realizadas. Como o ensaio não fornece o valor da função fluência no tempo zero, um ajuste exponencial simples foi adotado para extrapolar os dados e calcular esse valor. Além disso, o módulo de Young instantâneo foi calculado a partir do mesmo mecanismo, e o módulo de Young em longo prazo foi adotado como sendo o módulo de relaxação ao final do ensaio, quando este alcança um platô de estabilização final.

Para estabelecer a comparação entre os resultados numéricos e experimentais foi determinado a média do erro relativo. $\mathrm{O}$ valor do erro foi determinado no tempo, adotando como valor de referência o deslocamento experimental. Para o cálculo foram desconsiderados os valores correspondentes aos primeiros 10 s do ensaio, já que durante este período existe muito ruído nos resultados. O ruído nos tempos iniciais é ocasionado, provavelmente, pela ocorrência do rearranjo interno das partículas, assim como, da impossibilidade do equipamento atingir imediatamente o valor de pressão de $100 \mathrm{kPa}$ requerido no ensaio.

\section{Resultados}

Neste item são apresentados os resultados das simulações numéricas durante o período do carregamento do ensaio de fluência estática. As simulações correspondem a fase do carregamento no ensaio. O modelo viscoelástico exibe dificuldades para representar a fase do descarregamento como mostrado no trabalho de Rodrigues et al. (2016). Uma possível solução seria a programação de uma UMAT (User Material) para implementar um modelo constitutivo que mostre a parcela plástica do comportamento das misturas asfálticas. No entanto, o objetivo do trabalho tem como foco a modelação viscoelástica das misturas asfálticas. Utilizou-se como variável de controle o deslocamento vertical no tempo e comparou-se os resultados numéricos com os experimentais.

\begin{tabular}{|c|c|c|c|c|c|c|c|}
\hline \multicolumn{8}{|c|}{ Mistura: 4\% CAP 50-70 Modificado } \\
\hline \multicolumn{4}{|c|}{ Quase elástica } & \multicolumn{4}{|c|}{ Interconversão } \\
\hline \multicolumn{2}{|c|}{ Fluência $D(t)$} & \multicolumn{2}{|c|}{ Relaxação E(t) } & \multicolumn{2}{|c|}{ Fluência D(t) } & \multicolumn{2}{|c|}{ Relaxação E(t) } \\
\hline $\mathbf{D}_{0}$ & 0,004715 & $\mathbf{E}_{\infty}$ & 7,10 & $\mathbf{D}_{0}$ & 0,0045 & $\mathbf{E}_{\infty}$ & 7,07 \\
\hline$\tau_{\mathbf{i}}$ & $\mathbf{D}_{\mathbf{i}}$ & $\rho_{\mathrm{i}}$ & $\mathbf{E}_{\mathbf{i}}$ & $\tau_{\mathbf{i}}$ & $\mathbf{D}_{\mathbf{i}}$ & $\rho_{\mathbf{i}}$ & $\mathbf{E}_{\mathbf{i}}$ \\
\hline 1 & 0,00135 & 1 & 143,27 & 1 & 0,0016 & 1 & 175,24 \\
\hline 10 & 0,033 & 10 & 35,57 & 10 & 0,0329 & 10 & 10,32 \\
\hline 100 & 0,062 & 100 & 10,49 & 100 & 0,0622 & 100 & 5,91 \\
\hline 1000 & 0,04 & 1000 & 2,25 & 1000 & 0,0401 & 1000 & 1,56 \\
\hline
\end{tabular}

Tabela 1. Parâmetros da série de Prony para as misturas asfálticas analisadas 
Quase elástica

\begin{tabular}{|c|c|c|c|c|c|c|c|}
\hline \multicolumn{2}{|c|}{ Fluência D(t) } & \multicolumn{2}{|c|}{ Relaxação E(t) } & \multicolumn{2}{|c|}{ Fluência D(t) } & \multicolumn{2}{|c|}{ Relaxação E(t) } \\
\hline D0 & 0,00272 & $\mathbf{E}_{\infty}$ & 27,63 & D0 & 0,0025 & $\mathbf{E}_{\infty}$ & 25,36 \\
\hline$\tau_{\mathbf{i}}$ & $\mathbf{D}_{\mathrm{i}}$ & $\rho_{\mathrm{i}}$ & $\mathbf{E}_{\mathbf{i}}$ & $\tau_{\mathbf{i}}$ & $\mathbf{D}_{\mathbf{i}}$ & $\boldsymbol{\rho}_{\mathbf{i}}$ & $\mathbf{E}_{\mathbf{i}}$ \\
\hline 1 & 0,00172 & 1 & 212,57 & 3 & 0,00463 & 3 & 201,03 \\
\hline 10 & 0,00794 & 10 & 80,13 & 30 & 0,01075 & 30 & 23,78 \\
\hline 100 & 0,011 & 100 & 30,96 & 300 & 0,01102 & 300 & 15,70 \\
\hline 1000 & 0,013 & 1000 & 12,93 & 3000 & 0,01054 & 3000 & 6,06 \\
\hline
\end{tabular}

Tabela 2. Parâmetros da série de Prony para as misturas asfálticas analisadas (Continuação)

\section{Mistura: 5,5\% CAP 50-70}

Quase elástica

Interconversão

\begin{tabular}{|c|c|c|c|c|c|c|c|}
\hline \multicolumn{2}{|c|}{ Fluência D(t) } & \multicolumn{2}{|c|}{ Relaxação E(t) } & \multicolumn{2}{|c|}{ Fluência D(t) } & \multicolumn{2}{|c|}{ Relaxação E(t) } \\
\hline $\mathbf{D}_{\mathbf{0}}$ & 0,00215 & $\mathbf{E}_{\infty}$ & 26,11 & $\mathbf{D}_{\mathbf{0}}$ & 0,002 & $\mathbf{E}_{\infty}$ & 23,98 \\
\hline$\tau_{\mathbf{i}}$ & $\mathbf{D}_{\mathbf{i}}$ & $\rho_{\mathrm{i}}$ & $\mathbf{E}_{\mathbf{i}}$ & $\tau_{\mathbf{i}}$ & $\mathbf{D}_{\mathbf{i}}$ & $\rho_{\mathrm{i}}$ & $\mathbf{E}_{\mathrm{i}}$ \\
\hline 1 & 0,00137 & 1 & 294 & 3 & 0,00403 & 3 & 250,40 \\
\hline 10 & 0,00781 & 10 & 97,56 & 30 & 0,01136 & 30 & 23,91 \\
\hline 100 & 0,01300 & 100 & 35,13 & 300 & 0,01305 & 300 & 16,30 \\
\hline 1000 & 0,01400 & 1000 & 12,99 & 3000 & 0,01126 & 3000 & 5,63 \\
\hline \multicolumn{8}{|c|}{ Mistura: 6\% CAP 50-70 } \\
\hline \multicolumn{4}{|c|}{ Quase elástica } & \multicolumn{4}{|c|}{ Interconversão } \\
\hline \multicolumn{2}{|c|}{ Fluência D(t) } & \multicolumn{2}{|c|}{ Relaxação E(t) } & \multicolumn{2}{|c|}{ Fluência $\mathbf{D}(\mathbf{t})$} & \multicolumn{2}{|c|}{ Relaxação E(t) } \\
\hline $\mathbf{D}_{0}$ & $1,57 \mathrm{E}-03$ & $\mathbf{E}_{\infty}$ & 24,74 & $D_{0}$ & 0,0015 & $\mathbf{E}_{\infty}$ & 22,71 \\
\hline$\tau_{\mathbf{i}}$ & $\mathbf{D}_{\mathbf{i}}$ & $\rho_{\mathrm{i}}$ & $\mathbf{E}_{\mathrm{i}}$ & $\tau_{\mathbf{i}}$ & $\mathbf{D}_{\mathbf{i}}$ & $\rho_{\mathrm{i}}$ & $\mathbf{E}_{\mathrm{i}}$ \\
\hline 1 & 0,001029 & 1 & 449,23 & 3 & 0,003402 & 3 & 322,92 \\
\hline 10 & 0,00769 & 10 & 116,91 & 30 & 0,012028 & 30 & 20,14 \\
\hline 100 & 0,014 & 100 & 39,5 & 300 & 0,015031 & 300 & 16,90 \\
\hline 1000 & 0,016 & 1000 & 12,92 & 3000 & 0,01208 & 3000 & 5,25 \\
\hline
\end{tabular}

Nas Fig. 2; 3; 4 e 5 são mostrados os resultados do deslocamento tanto experimental como os obtidos nos modelos numéricos para cada mistura estudada. O deslocamento apresentado se refere a fase de carregamento durante o ensaio. Os gráficos sugerem que não existem diferenças notáveis entre os valores experimentais e as simulações. 


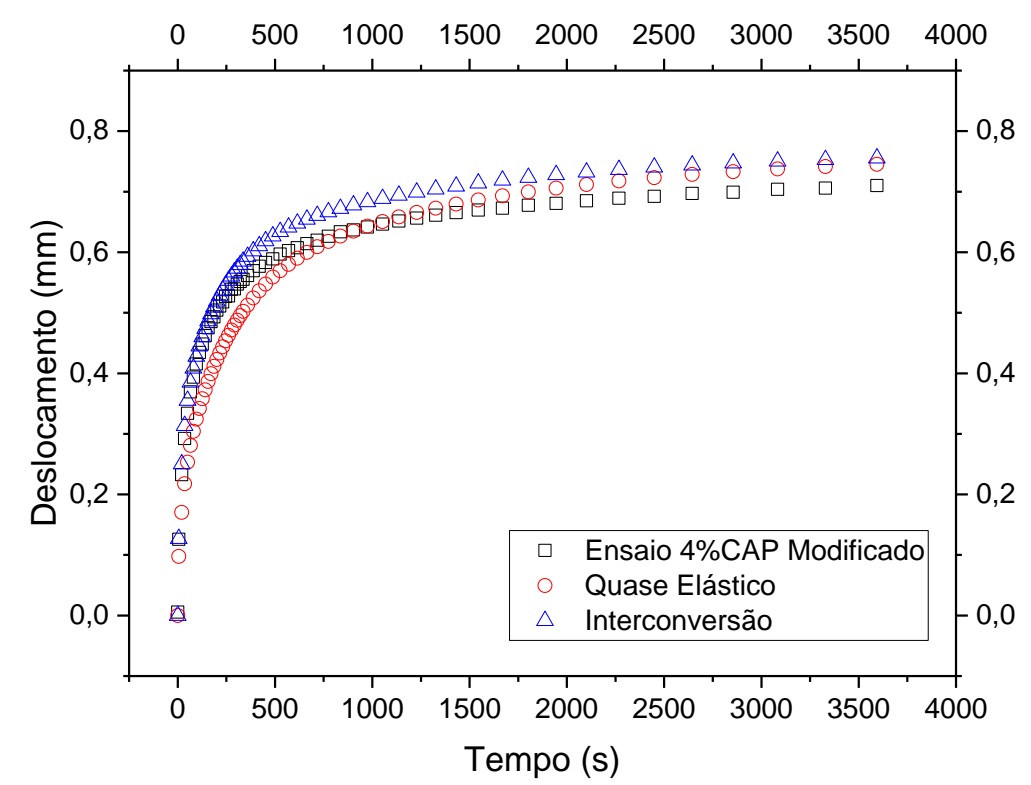

Figura 2. Resultados das simulações numéricas para misturas com 4\% de CAP 50-70 modificado com fibras.

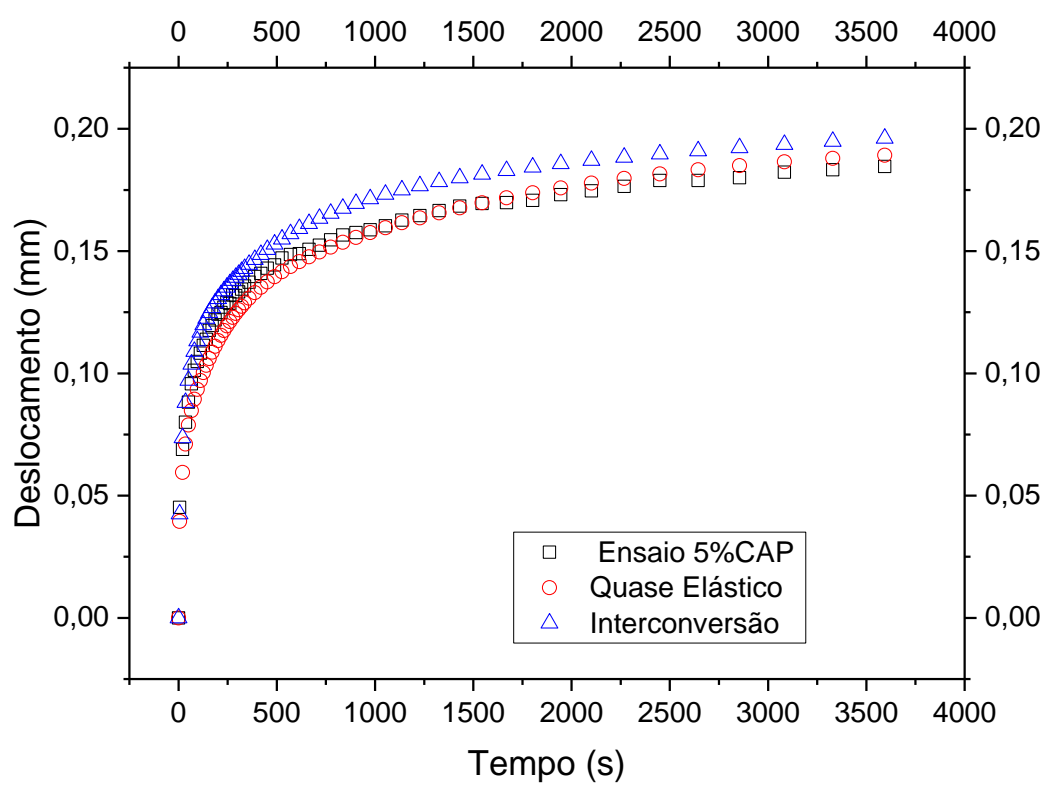

Figura 3. Resultados das simulações numéricas para misturas com $5 \%$ de CAP 50-70. 


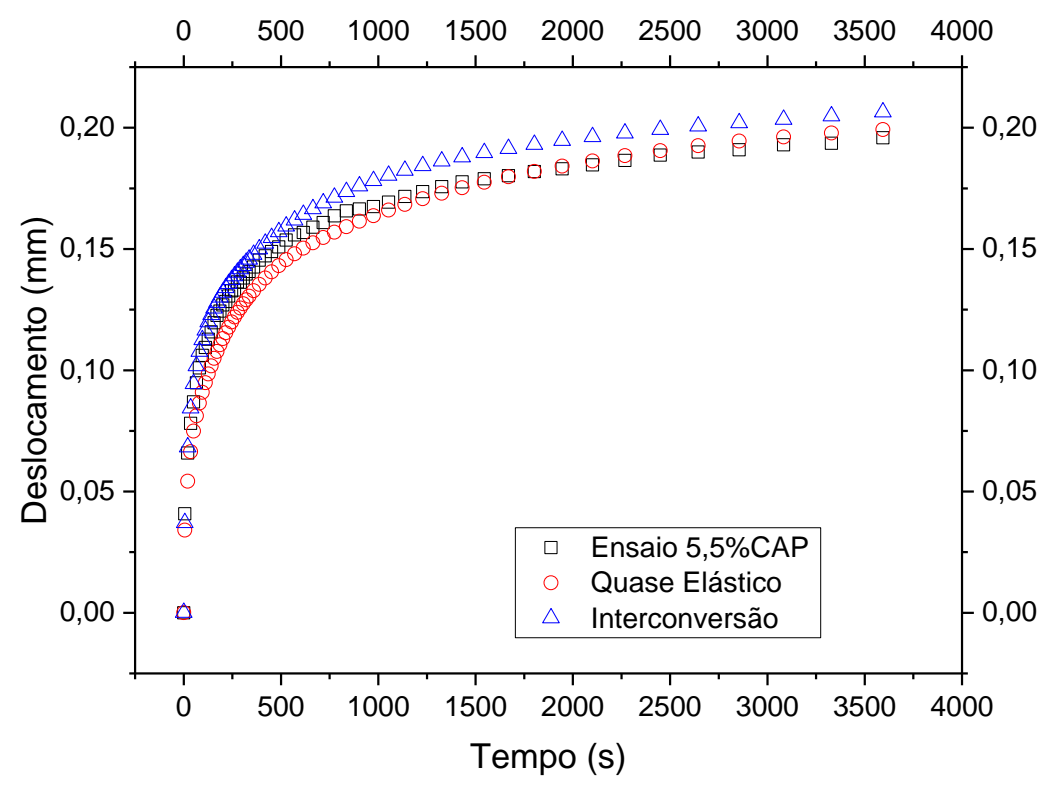

Figura 4. Resultados das simulações numéricas para misturas com 5,5\% de CAP 50-70.

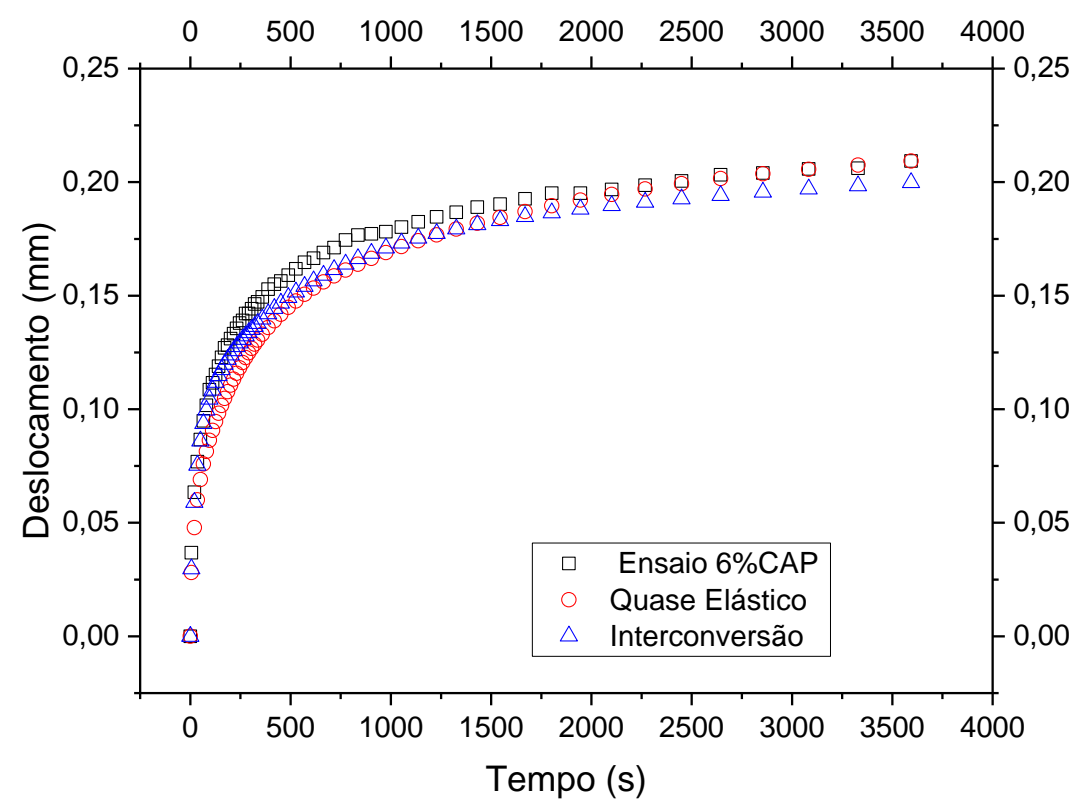

Figura 5. Resultados das simulações numéricas para misturas com 6\% de CAP 50-70.

$\mathrm{NaTab}$

Tabela 3 é apresentado o valor da média do erro relativo entre as simulações numéricas e os valores experimentais. Para as misturas estudadas a média do erro relativo não ultrapassa $10 \%$ comparando os valores experimentais com as simulações numéricas. Os resultados mostram que existe pouca influência na escolha da interconversão ou a relação quase elástica na determinação dos parâmetros constitutivos. Esta pouca influência é mantida independentemente do tipo e do teor de ligante asfáltico que apresentaram os compósitos estudados.

Tabela 3. Média do Erro relativo

\begin{tabular}{ccc}
\hline Mistura Asfáltica & Modelo Numérico & $\begin{array}{c}\text { Média do Erro } \\
\text { Relativo (\%) }\end{array}$ \\
\hline
\end{tabular}




\begin{tabular}{lcl}
\hline \multirow{2}{*}{ CPA 4\% CAP MOD } & Quase elástica & 6,1 \\
& Interconversão & 6,31 \\
\hline \multirow{2}{*}{ CPA 5\% CAP 50-70 } & Quase elástica & 8,31 \\
& Interconversão & 6,71 \\
\hline \multirow{2}{*}{ CPA 5,5\% CAP 50-70 } & Quase elástica & 5,78 \\
& Interconversão & 5,33 \\
\hline \multirow{2}{*}{ CPA 6\% CAP 50-70 } & Quase elástica & 4,05 \\
& Interconversão & 6,68 \\
\hline
\end{tabular}

\section{Considerações finais}

Foram utilizados dois métodos para determinar os parâmetros constitutivos viscoelásticos das misturas asfálticas do tipo CPA. No primeiro caso foi utilizado o método da Colocação por Mínimos Quadrados Linear (MQL), visando ajustar com a série de Prony as funções fluência e módulo de relaxação. Neste caso o módulo de relaxação foi obtido através da relação quase elástica. No segundo caso os parâmetros foram determinados com a interconversão da função fluência e o módulo de relaxação e com a ajuda do software VISCOlab ${ }^{\mathrm{TM}}$.

Os resultados numéricos mostram a pouca influência da escolha do método para obter os parâmetros constitutivos. Através da determinação da média do erro relativo entre as simulações numéricas e os valores experimentais e possível quantificar esta pouca influência. Os valores de média do erro relativo resultam num valor inferior ao $10 \%$ para todos os casos. Os resultados numéricos exibem praticamente o mesmo valor de acurácia com respeito aos valores experimentais o que demostra a pouca influência do método na determinação dos parâmetros constitutivos nos compósitos estudados.

\section{Referências}

1. Amaral, S. D. C. (2000). Estudos De Misturas Asfálticas Densas Com Agregados Do Estado Do Pará, Utilizando Asfalto Convencional (Cap-40) E Asfalto Modificado Com Polímero Sbs (Betuflex B 65/60), 147.

2. Brinson, H. F., \& Brinson, L. C. (2008). Polymer engineering science and viscoelasticity: An introduction. Polymer Engineering Science and Viscoelasticity: An Introduction (Second Edi). New York: Springer Science. https://doi.org/10.1007/978-0-387-73861-1

3. Collop, A., McDowell, G., \& Lee, Y. (2007). On the Use of Discrete Element Modeling to Simulate the Viscoelastic Deformation Behaviour of an Idealized Asphalt Mixture. Geomech Geoeng, 2(2), 77-86.

4. Evangelista Jr., F. (2006). Análise quasi-estática e dinâmica de pavimentos asfálticos. Universidade Federal do Ceará.

5. Evangelista Jr., F., Parente Jr., E., \& Barbosa S., J. (2005). Viscoelastic and elastic structural analysis of flexible pavements. Cilamce 2005, (October), $15 . \quad$ Retrieved from http://repositorio.ufc.br/bitstream/riufc/5666/1/2005_eve_eparentejunior.pdf

6. Fernández, F. P., Rodríguez, A. D., Lamela, R. M. J., \& Fernández, C. A. (2009). Estudio de la Interconversión entre las Funciones de Comportamiento de un Material Viscoelástico. Anales de Mecánica de La Fractura, 1(26), 244249. Retrieved from www.gef.es/Congresos/26/pdf/40.pdf

7. Fernández, P., Rodríguez, D., Lamela, M. J., \& Fernández-Canteli, A. (2011). Study of the interconversion between viscoelastic behaviour functions of PMMA. Mechanics of Time-Dependent Materials, 15(2), $169-180$. https://doi.org/10.1007/s11043-010-9128-3

8. Huang, Y. H. (1993). Pavement Analysis and Desing. New Jersey: Prentice-Hall.

9. Kim, Y.-R. (2013). Multiscale Modeling of Damage Evolution in Viscoelastic Bituminous Mixtures Subjected to Cyclic Loading. Journal of Engineering Materials and Technology, 135(2), 0210051-0210059. https://doi.org/10.1115/1.4023773

10. Kim, Y. R. (2009). Modeling of Asphalt Concret. (McGraw-Hill, Ed.). North Carolina.

11. Kong, J., \& Yuan, J. Y. (2010). Application of linear viscoelastic differential constitutive equation in ABAQUS. International Conference on Computer Design and Applications, ICCDA 2010, 5(Iccda), $152-156$. https://doi.org/10.1109/ICCDA.2010.5541456 
12. Park, S. W., \& Kim, Y. R. (1999). Interconversion between relaxation modulus and creep compliance for viscoelastic solids. MATERIALS IN CIVIL ENGINEERING, (February), 76-82.

13. Park, S. W., \& Schapery, R. A. (1999). Methods of interconversion between linear viscoelastic material functions. Part I-a numerical method based on Prony series. International Journal of Solids and Structures, 36(11), 16531675. https://doi.org/10.1016/S0020-7683(98)00055-9

14. Rodrigues, I. A., García, L. M., Muniz, M. F., \& Rodrigues, L. G. (2016). Simulação Numérica de Ensaios de Fluência Estática em Areia Asfalto. In $45^{\circ}$ Reunião Anual de Pavimentação - $19^{\circ}$ Encontro Nacional da Conservação Rodoviária (p. 13). Brasilia: ABDER-ABPv-DER/DF. Retrieved from http://www.rapvenacor.com.br/anais/Ordem 60 Artigo 10034.pdf

15. Silva, H. N. (2009). Caracterização Viscoelástica linear de misturas asfálticas : Operacionalização Computacional e Análise pelo Método dos Elementos Finitos. Universidade Federal do Ceará.

16. Silva, H. N., Sousa, P. C., Holanda, Á., \& Soares, J. B. (2008). A computer program for linear viscoelastic characterization using prony series.

17. Sousa, P. C., \& Soares, J. B. (2007). Método da colocação para obtenção de séries de pro y usadas a caracterização viscoelástica de materiais asfálticos.

18. Souza, F. V. de, \& Soares, J. B. (2002). Previsão do comportamento mecânico de misturas asfálticas a partir dos resultados do ensaio de Creep estático. Anais Do XVI Congresso de Pesquisa e Ensino Em Transportes, ANPET, Natal, (2001).

19. Souza, M. V. R. (2010). Efeito de fibras sintéticas nas propriedades de ligantes e concretos asfalticos. Universidade de Brasília. 